\title{
Calidad de la atención en el servicio de anestesiología. Percepción del usuario
}

\author{
Cristhofer Abel Torres-González, ${ }^{1}$ Yolanda Valera-Rodríguez, ${ }^{1}$ Elena Pinto Segura-María ${ }^{1}$
}

\begin{abstract}
Resumen
Antecedentes: La evaluación de la atención por los pacientes es esencial para la mejora de la calidad anestésica. Objetivo: Conocer la percepción de la calidad de la atención anestésica en nuestro hospital. Pacientes y métodos: Adultos sin compromiso mental o neurológico. Se utilizó un cuestionario diseñado por nosotros (Cuestionario de Percepción de Calidad Anestésica, CPCA) con dos dominios (comunicación y eficacia) y una pregunta de satisfacción general. Resultados: De 103 individuos, la percepción global fue excelente en $64 \%$, buena en $33.9 \%$, regular en un individuo y mala en otro. La comunicación fue percibida por el $96.2 \%$ de los sujetos como buena a excelente y por $3.8 \%$ (4/103) como muy mala. En cuanto a eficacia, $67.9 \%$ la percibió de buena o excelente, $26 \%$ como regular y $5.8 \%$ como mala. La mediana de puntuación de la satisfacción fue 8 (10 puntos máximo). Las principales causas para una mala percepción en la comunicación fueron una escasa información sobre los riesgos anestésicos y no identificar al anestesiólogo responsable. Para la calidad, la mayor insatisfacción fue la presencia de dolor postanestésico. Conclusión: Nuestra atención anestésica fue percibida con altos niveles de satisfacción. Es necesario mejorar la comunicación de los riesgos anestésicos y el control del dolor postcirugía.
\end{abstract}

Palabras clave: Percepción del paciente, evaluación preanestésica, satisfacción, comunicación.

\footnotetext{
1 Facultad Mexicana de Medicina, Universidad La Salle y Hospital Ángeles Clínica Londres.
}

Correspondencia:

Cristhofer Torres González

Correo electrónico: kris_30233@hotmail.com

Aceptado: 04-05-2016.

Este artículo puede ser consultado en versión completa en http:// www.medigraphic.com/actamedica

\begin{abstract}
Summary
Background: The evaluation of the patient satisfaction is essential to improve the quality of anesthesia. Objective: To know the perception of patients regarding the quality of the anesthesia received at our hospital. Patients and methods: Adults without mental or psychiatric impairment. We applied a questionnaire designed by us (Cuestionario de Percepción de Calidad Anestésica, CPCA) with two domains (communication and anesthesia efficacy) and patient's overall satisfaction. Results: 103 patients. The overall satisfaction was excellent in $64 \%$, good in $33.9 \%$, regular in one person and bad in another. In the communication domain, $96.2 \%$ perceived it either good or excellent and 3.8\% (4/103) as very bad. For efficacy, 67.9\% perceived it either good or excellent, $26 \%$ regular and $5.8 \%$ bad. The median of the satisfaction score was 8 (maximum 10 points). The main reasons for the perception of a bad communication were little information anesthesia risks or/and failure on an introduction by the anesthesiologist. Concerning anesthesia efficacy, the principal factor for dissatisfaction was the presence of post-anesthetic pain. Conclusion: A high proportion of our patients expressed satisfaction with the anesthesia received. To improve, a better communication of the anesthetic risks is necessary, as well as control of post-surgical pain.
\end{abstract}

Key words: Patient perception, anesthetic evaluation, satisfaction, communication quality.

\section{INTRODUCCIÓN}

La evaluación de la calidad de la atención médica es necesaria para mejorar los servicios prestados a los pacientes. ${ }^{1}$ Aunque existen diversos métodos para ello, una parte sustancial a considerar es la satisfacción desde la perspectiva del usuario. ${ }^{2}$ Esta condición, aunque difícil de medir, ${ }^{1}$ informa sobre el proceso recibido durante su atención y puede ser la base para una futura recomendación para otros pacientes en la decisión de acudir o no a un centro médico en particular. Por ello, instituciones como la nuestra la consideran muy trascendente. 
La medición de la satisfacción de la atención anestésica en los pacientes sometidos a procedimientos quirúrgicos ha empezado a cobrar importancia apenas en años recientes $^{3}$ por dos motivos principales: primero, porque es difícil aislarla del impacto de todo el proceso de atención quirúrgica, y, en segundo lugar, por la complejidad para medirla a través de cuestionarios. ${ }^{1}$ Sin embargo, diversos autores han señalado que una mala atención anestésica repercute significativamente en la satisfacción final de un individuo sometido a un proceso quirúrgico. ${ }^{4}$

Para evaluar la percepción de satisfacción del sujeto sobre la anestesia recibida se han usado diferentes instrumentos. ${ }^{1,5}$ Estos suelen explorar tres áreas relacionadas con la calidad. La primera es sobre la comunicación y relación paciente-anestesiólogo; en ella se evalúa la información otorgada con respecto a la comprensión del individuo sobre la anestesia a recibir y sus riesgos, así como la empatía y confianza ganada por el anestesiólogo. ${ }^{5}$ La segunda, no siempre evaluada, es la calidad de la anestesia en cuanto a su eficiencia, es decir, si la anestesia logró controlar el dolor. En este rubro, cuando se otorgó una anestesia general, se investiga si el sujeto presentó despertares o sensación de no estar dormido durante el procedimiento quirúrgico o si sintió dolor; para la anestesia local-regional, las peguntas van más dirigidas a si tuvo alguna sensación de dolor durante el procedimiento quirúrgico. ${ }^{6}$ La tercera área evaluada se dirige al control de síntomas en el postoperatorio; de ellos, el más importante es el dolor, seguido de náuseas, vómitos, cefalea, escalofríos, disfonía, sed y mareos, entre los más comúnmente referidos. ${ }^{1,5}$
Con estas evaluaciones, diferentes estudios internacionales han informado, en general, niveles de satisfacción por arriba del $80 \%{ }^{1,5}$

En nuestro hospital, como parte del programa de mejora en la calidad de la atención, se han realizado diferentes mediciones, pero este es el primer trabajo sobre la satisfacción percibida por los pacientes sobre la atención anestésica.

\section{MATERIAL Y MÉTODOS}

El estudio se llevó a cabo en el Hospital Ángeles de la Clínica Londres. Se realizó con base en los términos de las declaraciones de Helsinki y Ginebra de la Asociación Médica Mundial y en acuerdo con lo estipulado en el Reglamento de la Ley General de Salud en Materia de Investigación para la Salud, así como con previa aprobación por el Comité de Ética del hospital y consentimiento informado por cada uno de los participantes.

Cuestionario de Percepción de Calidad Anestésica (CPCA). Este fue realizado con base en una adaptación del "Quality of Recovery Score, QoR". ${ }^{7}$ Un grupo de expertos (MEPS, HCS, YVR y EGS) por consenso adecuaron las preguntas al tipo de individuos atendidos en nuestro hospital para generar una versión corta y sencilla de aplicar. Asimismo, escogieron las mejores peguntas para evaluar la comunicación y la eficacia (Cuadro 1). El cuestionario fue validado en sus dominios (contenido) por medio de un análisis de componentes principales y método de rotación normalización varimax de Kaiser. La evaluación de su consistencia interna se hizo con el alfa de Conbrach por el método de K-20 de Richardson. ${ }^{8}$

\section{Cuadro 1. Cuestionario de percepción de calidad anestésica (CPCA).}

Pregunta 1. ¿El anestesiólogo lo visitó antes de la cirugía?

Pregunta 2. ¿Considera que le explicó adecuadamente el procedimiento anestésico?

Pregunta 3. Dentro de la información que le dio acerca del procedimiento anestésico, ¿le mencionó los riegos del mismo?

Pregunta 4. ¿Considera que la explicación ofrecida fue bien comprendida por usted?

Pregunta 5. ¿Resolvió el anestesiólogo todas sus dudas sobre la anestesia?

Pregunta 6. ¿Sintió dolor después de su anestesia?

Pregunta 7. ¿Presentó alguna molestia durante la atención anestésica? Anotar de las siguientes (puede ser más de una): náusea, vómito o cefalea

Pregunta 8. ¿Quedó satisfecho con el servicio de anestesia?

Evaluación general: ¿Cómo consideró la atención que le otorgó el anestesiólogo?
Mala (
) Regular (
) Buena (
) Excelente (
)

A cada pregunta conteste "si" o "no".

Preguntas 1 a 6 y 8 : un punto por cada "Si"

Pregunta 7: un punto por cada síntoma referido.

El puntaje máximo es 10. 
Pacientes. Participaron sujetos mayores de 18 años sometidos a una cirugía de tipo electiva o de urgencia bajo anestesia general o regional que tuvieron la capacidad mental para aprobar su cirugía. Después de sus 24 horas postquirúrgicas, se les invitó a contestar nuestro cuestionario aplicado cara a cara. Por conveniencia, se incluyó a quienes aceptaron durante el periodo del 1 de septiembre al 30 de noviembre de 2015.

Método. Las personas fueron entrevistadas antes de su egreso. El cuestionario fue aplicado por uno de los investigadores capacitados para ello. Durante la entrevista, se resolvió cualquier duda sin influir en las respuestas emitidas por los individuos.

Análisis estadístico. Se obtuvieron las frecuencias simples y porcentajes para las variables cualitativas y las medias con sus desviaciones estándar para las cuantitativas. La satisfacción de la atención fue evaluada, primero, con la percepción emitida por los pacientes en su valoración general y expresada como excelente, buena, regular y mala (pregunta 8).

En segundo lugar, se tomó en cuenta la puntuación obtenida por el CPCA, la cual se calificó por dos dominios: la comunicación (preguntas 1 a 5), interpretada como excelente (5 puntos), muy buena (4), buena (3), regular (2), mala (1) y muy mala (cero), y la eficacia (preguntas 6 y 7), interpretada como excelente (4 puntos), buena (3), regular (2), mala (1) y muy mala (cero). Finalmente, se adicionó un punto más si el sujeto informó "estar satisfecho" con el procedimiento. La puntuación máxima de satisfacción de la escala fue "10" y la total insatisfacción, " 0 ". Por último, se realizó una correlación de Rho de Spearman entre la percepción general de la evaluación general de la atención otorgada contra el puntaje de CPCA. Todos los análisis se llevaron a cabo con el paquete estadístico SPSS versión 20. La significancia estadística de la correlación se aceptó con una $\mathrm{p}<0.05$.

\section{RESULTADOS}

Validación del cuestionario. Los expertos apoyaron la versión final (validez de apariencia). La validez de contenido mostró por análisis factorial dos dominios principales con autovalores de 3.6 (dominio 1) y 1.76 (dominio 2). En conjunto, estos dos valores explicaron el $77.3 \%$ de la varianza (dominio $1=52.1 \%$, dominio $2=25.2 \%$ ). En el dominio 1, cargaron las preguntas 1 (0.98), 2 (0.93), 3 (0.98), 4 (0.61) y 5 (0.62); para el dominio 2 , cargaron las preguntas $6(0.85), 7(0.73)$ y $8(0.80)$. Por tanto, el dominio 1 se definió como "comunicación" y el dos como "eficacia". La escala mostró una confiabilidad interna con alfa de Cronbach de 0.70.

Pacientes. Se obtuvo una muestra de 103 participantes. Como se muestra en el cuadro 2, hubo un leve predominio de mujeres y la edad promedio fue de 48 años; asimismo, la mayoría recibieron anestesia general.

Evaluación general de la calidad de la atención anestésica. Como se muestra en la figura 1, a la pregunta expresa, la mayoría de las personas la consideró como de buena a excelente (101/103 o 98\%). El individuo que la calificó como mala informó no haber tenido contacto con el anestesiólogo tratante antes de la cirugía.

Evaluación de la calidad de la anestesia por el cuestionario CPCA. Esta fue analizada, primero, en cada uno de sus dominios y luego, de manera general. En el dominio de comunicación (Figura 2 y Cuadro 3), se observó que el $87.4 \%(90 / 103)$ de los sujetos la calificaron de muy buena a excelente. Los principales problemas para una insatisfacción fueron, en primer lugar, la falta de información sobre los riesgos de la anestesia, la cual sucedió en el 38.8\% de los pacientes; el segundo aspecto se relacionó con

\begin{tabular}{|c|c|c|}
\hline \multicolumn{3}{|c|}{$\begin{array}{c}\text { Cuadro 2. Características de los pacientes evaluados } \\
\text { en la calidad de su anestesia }(n=103) .\end{array}$} \\
\hline Dato & & n (\%) \\
\hline \multirow[t]{2}{*}{ Sexo } & Masculinos & $42(40.8)$ \\
\hline & Femeninos & $61(59.2)$ \\
\hline Edad en años & Media (1 DE) & $48(17)$ \\
\hline \multirow[t]{2}{*}{ Tipo de anestesia } & General & $83(77.7)$ \\
\hline & Regional & $20(22.3)$ \\
\hline
\end{tabular}

Figura 1. Percepción general de los individuos en relación con la calidad de la anestesia recibida $(n=103)$.

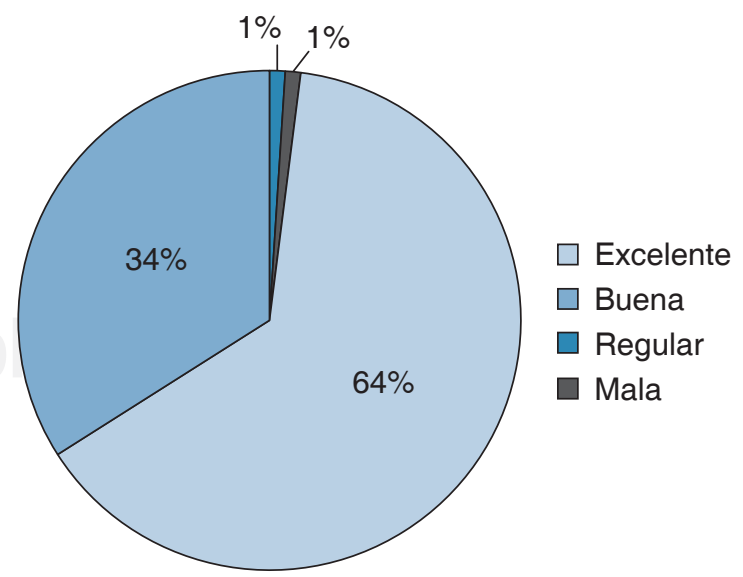

Respuesta a la evaluación general: ¿Cómo consideró la atención que le otorgó el anestesiólogo? 
la percepción de las personas de falta de claridad en las explicaciones. Los cuatro sujetos (3.9\%) que la calificaron como muy mala, refirieron no haber tenido contacto con el médico anestesiólogo tratante antes de su cirugía.

Con respecto a la evaluación de la satisfacción de la anestesia en su eficacia (Cuadro 4 y Figura 2), el 67.9\% (70/103) de los individuos la calificaron de excelente o buena por no tener síntomas o sólo uno (Figura 3). El resto la puntuaron como baja, sobre todo porque mencionaron haber tenido dolor postquirúrgico. Asimismo, cinco pacientes tuvieron tres síntomas y, por tanto, la calificaron como muy mala.

Figura 2. Evaluación de la dimensión "comunicación efectiva" por los pacientes en el "Cuestionario de percepción de calidad anestésica” (CPCA).

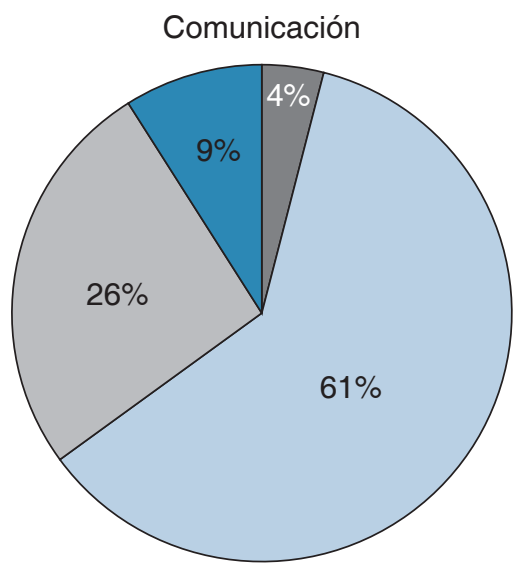

$\square$ Excelente (5 puntos)

Buena (3 puntos) $\square$ Muy buena (4 puntos)

$\square$ Muy mala (0 puntos)
En la puntuación general (Figura 4), la escala CPCA permitió determinar que el 90\% de los sujetos (93/103) calificó la anestesia como satisfactoria, con una puntuación de 8 o más. Cinco personas la evaluaron como regular (puntaje de 6 a 7) y cinco como mala o reprobatoria (puntaje de 4 a 5).

Por último, al analizar si había correlación entre el puntaje emitido por el CPAC y la respuesta a la percepción general, observamos que no fue buena (Rho Spearman $=0.48, \mathrm{p}<0.001)$. Parte de la discrepancia se debió a una aseveración de alta satisfacción a la pregunta expresa, pero una mala calificación anotada en el CPCA, hecho observado en nueve individuos (Cuadro 5).

\section{DISCUSIÓN}

Este es el primer estudio sobre la percepción de la satisfacción anestésica realizado en nuestro hospital; de acuerdo con nuestros resultados, encontramos una alta percepción de los pacientes como buena a excelente con base en los puntajes emitidos en el CPCA y en la evaluación general.

Como se comentó en los antecedentes, la evaluación de la satisfacción anestésica es difícil por no existir un instrumento considerado como estándar de oro aceptado mundialmente. ${ }^{1}$ Por ello, nosotros utilizamos una adaptación de uno de los más reconocidos y empleados en estudios previos, el QoR40.7 En la adaptación que realizamos, incorporamos la metodología requerida para validar nuestro instrumento; esto es, primero hicimos una adaptación corta en contenido y claridad con la ayuda de un panel de expertos; después, confirmamos la validez de contenido a través de un análisis de componentes principales. Este no corroboró la existencia de las dos dimensiones que nos interesaba evaluar (comunicación y eficacia). Asimismo, por

\begin{tabular}{|c|c|c|c|}
\hline \multicolumn{4}{|c|}{$\begin{array}{l}\text { Cuadro 3. Respuestas otorgadas al "Cuestionario de percepción de calidad anestésica" } \\
\text { (CPCA) relacionadas a la comunicación }(n=103) \text {. }\end{array}$} \\
\hline & & Respuesta afirmativa & Respuesta negativa \\
\hline Núm. & Pregunta & n $(\%)$ & n $(\%)$ \\
\hline 1. & ¿El anestesiólogo lo visitó antes de la cirugía? & $99(96.1)$ & $4(3.9)$ \\
\hline 2. & $\begin{array}{l}\text { ¿Considera que en esta visita le explicó } \\
\text { adecuadamente el procedimiento anestésico? }\end{array}$ & $99(96.1)$ & $4(3.9)$ \\
\hline 3. & $\begin{array}{l}\text { Dentro de la información que le dio de la anestesia, } \\
\text { ¿le mencionó los riesgos de la misma? }\end{array}$ & $63(61.2)$ & $40(38.8)$ \\
\hline 4. & $\begin{array}{l}\text { ¿Considera que la explicación ofrecida fue bien } \\
\text { entendida por usted? }\end{array}$ & 90 (87.4) & $13(12.6)$ \\
\hline 5. & $\begin{array}{l}\text { ¿Le resolvió el anestesiólogo todas sus dudas } \\
\text { sobre la anestesia? }\end{array}$ & $99(96.1)$ & $4(3.9)$ \\
\hline
\end{tabular}




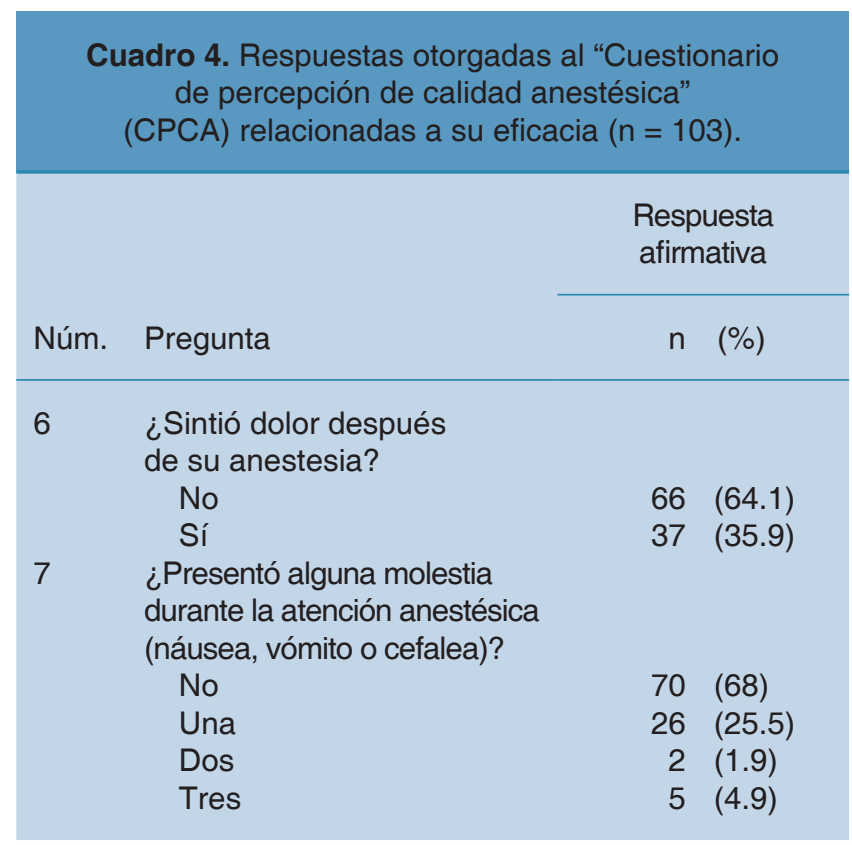

Figura 3. Evaluación de la dimensión "anestesia efectiva" por los sujetos en el "Cuestionario de percepción de calidad anestésica” (CPCA) ( $n=103)$.

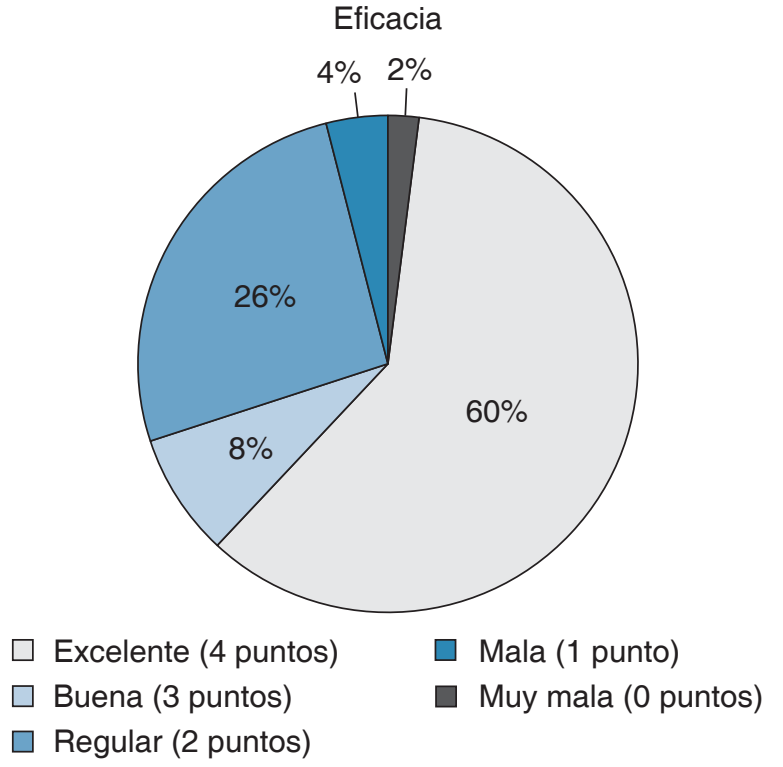

medio del análisis de alfa de Conbrach, pudimos demostrar una adecuada confiabilidad (alfa de 0.70).6,8

Por medio de nuestro instrumento (CPCA), pudimos establecer la satisfacción, tanto general como en las dos dimensiones más importantes y consideradas en otros estudios: la comunicación y la eficacia. Con respecto a la satisfacción en la atención medida por la comunicación, encontramos que, en general, las personas se sintieron
Figura 4. Evaluación general en el "Cuestionario de la percepción de calidad anestésica” (CPCA).

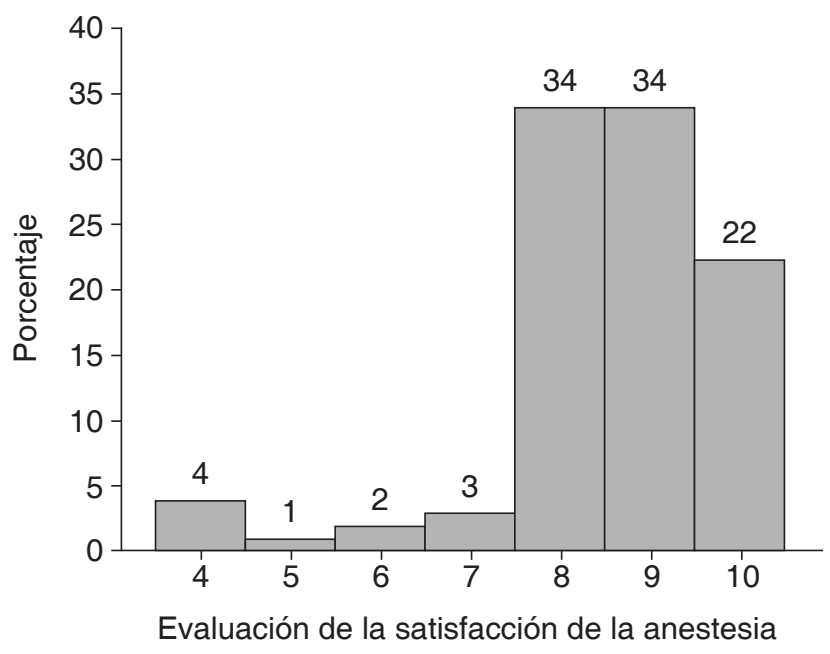

Escala de 10 puntos (5 de comunicación, 4 de eficacia y uno de satisfacción general [sí = 1, no = 0])

acompañadas e informadas sobre la atención anestésica recibida. Los pocos individuos que calificaron dicha satisfacción como baja justificaron su mala impresión por una interacción deficiente con el anestesiólogo tratante. Es importante resaltar que ningún sujeto entró a cirugía sin una valoración previa de un anestesiólogo; por ello, los pacientes que negaron la interacción se referían a no haber tenido un contacto con su anestesiólogo tratante, aunque sí lo tuvieron con el residente a cargo. Diversos instrumentos han considerado como una comunicación de alta calidad el otorgar una información clara tanto del procedimiento como de los riesgos inherentes al uso de anestésicos. ${ }^{1,5,6,9-11}$ Aunque es posible que algunas personas preferirían no escuchar los últimos porque les genera más angustia, esta situación puede repercutir en su satisfacción final. Se sabe que la información de los riesgos previene una insatisfacción ante complicaciones postanestésicas. ${ }^{6}$ Nuestro estudio no permitió establecer por qué no se les informaron los riesgos anestésicos a estos sujetos, pero sí nos indicó la necesidad de reforzar esta deficiencia.

En relación con la satisfacción de la eficacia anestésica al controlar el dolor trans- y postoperatorio, así como amnesia postoperatoria y sedación, nuestro instrumento se dirigió principalmente a la sintomatología postanestésica. Ello porque estudios previos han mencionado la baja frecuencia de eventos de despertares intraoperatorios y baja confiabilidad en su medición. ${ }^{1}$ Tal como en otros informes, ${ }^{4-6,11}$ en general, los individuos refirieron estar satisfechos al no haber tenido sintomatología postanestésica. Sin embargo, es muy difícil lograr que ningún sujeto 


Cuadro 5. Correlación entre la evaluación por el "Cuestionario de percepción
de calidad anestésica (CPCA)" con la evaluación general.

la presente. En nuestros pacientes, 32\% tuvieron al menos una. El síntoma que influye más sobre la satisfacción final es el dolor. Por ello, esta pregunta fue muy enfatizada en nuestro cuestionario (cerca de $36 \%$ lo presentaron). Como ya ha sido recomendado en otros trabajos, nosotros también insistimos en el control del dolor, dado que una persona mostrará mayor satisfacción en la atención si el anestesiólogo le resuelve su sensación dolorosa de manera pronta y permanente. ${ }^{1,5,12}$

En un estudio previo realizado en México en el año 2013 con individuos geriátricos, se encontraron similitudes con nuestro estudio y se hizo énfasis en la mejoría de la calidad percibida. ${ }^{13}$

Nuestra investigación tiene varias fortalezas. La primera es el cuidar aspectos importantes en la valoración de la satisfacción de los sujetos, tales como realizar la encuesta cuando el paciente está más consciente de su experiencia, utilizar un cuestionario consistente y con validez suficiente, aplicación de la encuesta con personal capacitado y ajeno a los objetivos del estudio, además de cara a cara. ${ }^{4}$ La segunda, fue el haber logrado un tamaño de muestra aceptable. Finalmente, la frecuencia de aceptación a la participación fue alta, ya que sólo cuatro personas invitadas la rechazaron, lo cual traduce una baja posibilidad de un sesgo de selección.

Por otro lado, varias limitaciones deben ser consideradas para la extrapolación de nuestros resultados: nuestro cuestionario cubre los dominios principales, pero podría ampliarse a la calidad transanestésica. Dado que esta es la primera vez que se aplica el CPCA, nuestros resultados no pueden ser comparados con los de otros estudios. La correlación entre el puntaje del CPCA y la pregunta general de evaluación de la calidad no mostró una alta correlación, lo cual puede representar diferencias en los aspectos medidos. Por lo anterior, es necesaria una validación por constructo con otros instrumentos.

Por el momento, recomendamos explorar la validez de nuestro instrumento con otros pacientes. Sin embargo, ante la ausencia de otra opción, consideramos que nuestro CPCA puede ser utilizado.

En conclusión, en esta muestra se encontró que más del $80 \%$ de los individuos expresaron una satisfacción de la anestesia recibida y la calificaron como buena a excelente. Las principales áreas de mejora son, para la comunicación, incrementar la información de los riesgos anestésicos de manera clara y con empatía suficiente para generar confianza, y para la eficacia, realizar acciones para evitar y atender con prontitud las molestias postoperatorias.

\section{REFERENCIAS}

1. Le May S, Hardy JF, Taillefer MC, Dupuis G. Patient satisfaction with anesthesia services. Can J Anesth. 2001; 48 (2): 153-161.

2. Heidegger T, Saal D, Nübling M. Patient satisfaction with anaesthesia. Part 1: satisfaction as part of outcome —and what satisfies patients. Anaesthesia. 2013; 68: 1165-1172.

3. Myles PS, Williams DL, Hendrata M, Anderson H, Weeks AM. Patient satisfaction after anaesthesia and surgery: results of a prospective survey of 10,811 patients. Br J Anaesth. 2000; 84: 6-10.

4. Bauer M, Böhrer H, Aichele G, Bach A, Martin E.. Measuring patient satisfaction with anaesthesia: perioperative questionnaire versus standardised face-to-face interview. Acta Anaesthesiol Scan. 2001; 45 (1): 65-72.

5. Heidegger T, Husemann $Y$, Nuebling M, Morf D, Sieber T, Huth A et al. Patient satisfaction with anesthesia care: development of a psychometric questionnaire and benchmarking among six hospitals in Switzerland and Austria. Br J Anaesth. 2002; 89 (6): 863-872. 
6. Hocking G, Weightman WM, Smith C, Gibbs NM, Sherrard K. Measuring the quality of anaesthesia from a patient's perspective: development, validation, and implementation of a short questionnaire. Br J Anaesth. 2013; 111 (6): 979-989.

7. Myles PS, Hunt JO, Nightingale CE, Fletcher H, Beh T, Tanil D. Development and psychometric testing of a quality of recovery score after general anesthesia and surgery in adults. Anesth Analg. 1999; 88 (1): 83-90.

8. Streiner DL, Norman GR. Health measurement scales: a practical guide to their development and use. Fifth edition, Oxford University press, London 2014.

9. Lagasse RS. Indicators of anesthesia safety and quality. Curr Opin Anaesth. 2007; 15 (2): 239-243.
10. Mahapatra P, Leong E. Improving documentation and communication using operative note proforms. BMJ Qual Improv Rep. 2016; 5 (1). pii: u209122.w3712.

11. Hadjistavropoulos HD, Dobson J, Boisvert JA. Information provision, patient involvement, and emotional support: prospective areas for improving anesthetic care. Can J Anesth. 2001; 48 (9): 864-870.

12. Bell DM, Halliburton JR, Preston JC. An evaluation of anesthesia patient satisfaction instruments. AANA J. 2004; 72: 211-217.

13. Castellanos A, Cervantes HE, Vásquez PI. Satisfacción anestésica como indicador de calidad de la atención médica en el paciente geriátrico. Revista Mexicana de Anestesiología. 2013; 36: 250-255. 